\title{
Direct synthesis of furan-2,5-dicarboxylic acid monoamides
}

\author{
Róbert Fischer* and Mária Fišerová \\ Pulp and Paper Research Institute, Lamačská cesta 3, 84104 Bratislava, Slovak Republic \\ E-mail: robofis@centrum.sk
}

DOI: $\underline{\text { http://dx.doi.org/10.3998/ark.5550190.p009.239 }}$

\begin{abstract}
A regioselective monoamidation of furan-2,5-dicarboxylic acid using $O$-(benzotriazol-1-yl)$N, N, N^{\prime}, N^{\prime}$-tetramethyluronium tetrafluoroborate is presented. The excellent regioselectivity in favour of activated monobenzotriazoyl ester as a intermediate is achieved by gradual addition of a coupling reagent into a dilute solution of furan-2,5-dicarboxylic acid and $N, N$ diisopropylethylamine in dimethylformamide. Divided crude reaction mixture is used directly in the subsequent coupling reactions with benzylamine, diethylamine, piperidine and aniline, respectively. Four representative monoamides are prepared and isolated in very good yields 73$82 \%$. Traces of symmetric diamides were also detected, however never in isolable amounts.
\end{abstract}

Keywords: Amidation, furan-2,5-dicarboxylic acid, TBTU, high value-added products

\section{Introduction}

An increasing demand for the production of organic compounds from renewable resources, as a suitable alternative to fuel-based raw materials, requires the development of new technological processes applicable in the synthesis of essential building blocks. ${ }^{1,2}$ The pre-extraction of hemicelluloses offers an interesting economic opportunity to the pulp and paper industry, ${ }^{3}$ affording chemicals that could be further converted into a wide variety of high value-added products. $^{4-6}$ Furan-2,5-dicarboxylic acid (2,5-FDCA, 1, Scheme 1) is a strategic chemical with high potential in the polymer industry. It is considered to be a excellent replacement for terephthalic acid, ${ }^{7}$ being a bio-based monomer precursor readily accessible from polysaccharides or sugars. New types of polymeric material derived from diesters of 2,5-FDCA exhibit qualitative properties comparable with poly(ethylene terephthalate). Moreover, they are more easily environmentally degradable. ${ }^{8-10}$ The production of $\mathbf{1}$ is usually based on processes including the conversion of hexoses under acidic conditions at higher temperatures ${ }^{10,11}$ and various oxidation reactions of 2,5-disubstituted furans, mainly 5-(hydroxymethyl)furfural. ${ }^{10,12,13}$ Recently, considerable attention has been paid to the production of 2,5-FDCA from $\mathrm{C}_{5}$ compounds. Furan-2-carboxylic acid and its salts have been successfully employed in Henkeltype reactions, affording furan and furan-2,5-dicarboxylate. ${ }^{14,15}$ Unfortunately, the direct use of $\mathbf{1}$ 
in manufacturing processes is rather impeded due to its low solubility in most industrial solvents. On the other hand, diesters of 2,5-FDCA are very soluble in organic solvents, and therefore more easily isolable. They are readily available by acid-catalyzed esterification of 2,5-FDCA in the corresponding alcohols. ${ }^{8,9,11,15,16}$ Oxidative esterification of 5-(hydroxymethyl)furan-2carbaldehyde by oxygen as well as air on gold catalysts, ${ }^{17,18}$ and the copper-catalyzed reaction of methyl furan-2-carboxylate with tetrachloromethane/methanol, ${ }^{19}$ are two modern methods. The reactions of 1 with diazomethane ${ }^{20}$ and with methanol in presence of $\mathrm{TMSCl}^{21}$ have also been published, but these are amongst the less convenient procedures.

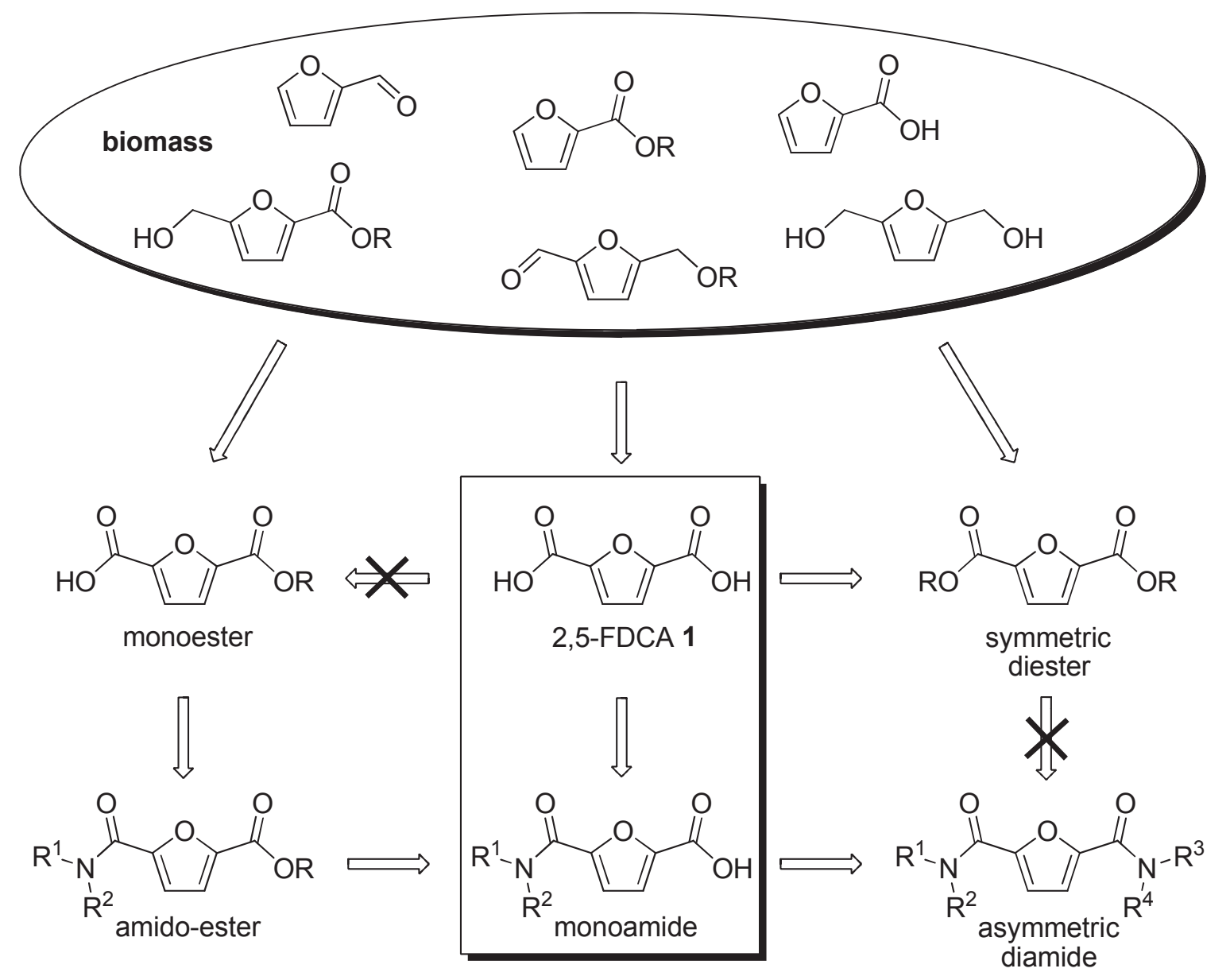

Scheme 1. Synthetic options for the preparation of asymmetric 2,5-FDCA diamides-high addedvalue products originating from renewable resources.

The monoesters of 2,5-FDCA are an interesting class of valuable 2,5-FDCA derivatives, often applied in various synthetic strategies. They are mainly prepared by oxidations of 5-(alkyloxymethyl)furan-2-carbaldehydes ${ }^{22-24}$ and methyl 5-(hydroxymethyl)furan-2-carboxylates, ${ }^{25}$ and by Henkel reaction of 2,5-FDCA with carbon dioxide in the corresponding alcohol. ${ }^{26}$ It is known that 2,5-FDCA and its derivatives are intermediates of interest in screening processes for 
pharmacological candidates. ${ }^{7,19,27}$ In particular, 2,5-FDCA diamides have been examined in studies of inhibitory activity against the cFMS tyrosine kinase as potential anti-inflammatory agents, ${ }^{28}$ in investigations for small-molecule ligands of methyl-lysine binding proteins ${ }^{29}$ and were also tested for their antiproliferative activity against human cancer cell lines, and for polynucleotide-binding activity. ${ }^{30}$ Such compounds have been prepared either directly from the corresponding dichloride, ${ }^{30}$ diester $^{31}$ and from 2,5-FDCA itself, ${ }^{29}$ (symmetric diamides) or by coupling reactions of suitably substituted monoamides, obtained from carbamoyl esters. ${ }^{25,29} \mathrm{~A}$ unique Pd-catalyzed aminocarbonylation of 5-bromosubstituted furan-2-carboxylic acid amide was reported to give an unsymmetrical diamide of 2,5-FDCA. ${ }^{32}$

\section{Results and Discussion}

Considering the high importance of 2,5-FDCA monoamides, we undertook a search for better and synthetically shorter methods for their preparation than the above-mentioned multistep procedures. In this paper, we report on a convenient regioselective monoamidation of $\mathbf{1}$, using TBTU 2 [O-(benzotriazol-1-yl)- $N, N, N^{\prime}, N^{\prime}$-tetramethyluronium tetrafluoroborate]. The use of $\mathrm{TBTU}^{33}$ as a highly potent amide coupling reagent is well-documented and has often been applied, mostly in medicinal chemistry. It is commercially available and can activate carboxyl groups under mild reaction conditions. The idea of selective monoamidation of symmetric aromatic and heteroaromatic dicarboxylic acids has attracted attention before; ${ }^{34-37}$ however, a general method is still lacking. To the best of our knowledge this is the first report of 2,5-FDCA monoamides being obtained directly and in good yields from the initial dicarboxylic acid.

In the search for optimal reaction conditions we systematically varied different reaction parameters, such the amount of TBTU, the speed of its addition, the excess of commonly recommended DIPEA ( $N, N$-diisopropylethylamine $),{ }^{38}$ the concentration of the reaction mixture, and the reaction temperature. Finally, we found that slow dropwise addition of TBTU solution in DMF at room temperature and a dilute reaction mixture resulted in regioselective reaction. A larger excess of DIPEA (3.5 equiv) and 1.2 equiv of TBTU seemed to be essential for the reaction completion (Scheme 2). It is noteworthy that TBTU activates a carboxylic group almost immediately to form $\mathbf{3}$.

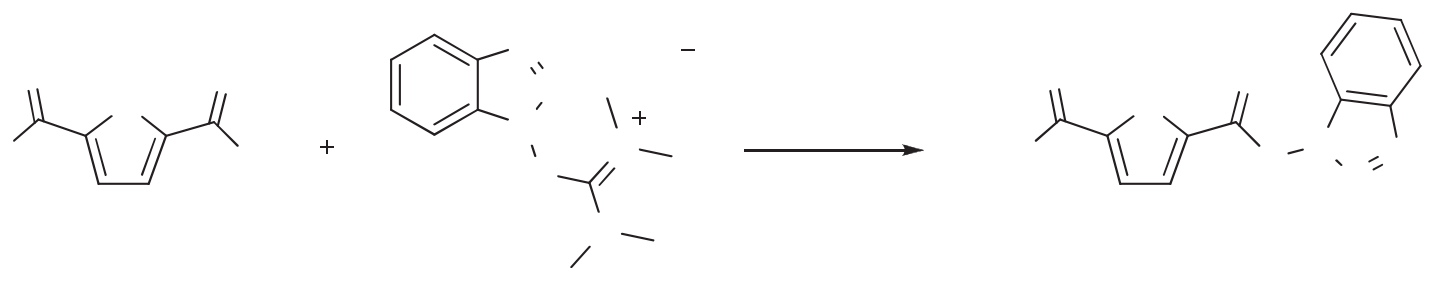

Scheme 2. Regioselective activation of 2,5-FDCA 1 with TBTU 2. 
The crude reaction mixture was used directly in the subsequent coupling reaction with an amine because of the expected instability of $\mathbf{3}$ during any separation process. For investigation we selected four representative amines, three aliphatic amines, benzylamine (4a), diethylamine (4b) and piperidine (4c), and aniline (4d) (Scheme 3). The nucleophile was added in one portion and the mixture was stirred at room temperature for 2 hours. After standard work-up, involving acidification with aqueous $\mathrm{HCl}$ and the extraction into ethyl acetate, the combined organic layers were washed with brine instead of water to reduce loss of the product from the acetate solution. The monoamides 5a-d were isolated by flash column chromatography on silica gel $\left(\mathrm{CH}_{2} \mathrm{Cl}_{2} / \mathrm{CH}_{3} \mathrm{OH} / \mathrm{CH}_{3} \mathrm{COOH}, 7: 1: 0.1\right)$ in good yields (73-82\%). Their structures were particularly assigned based on the multiplicity and coupling constants of the protons $\mathrm{H}-2$ and $\mathrm{H}-$ 3. In all cases we observed two doublets in the range $7.90-8.15$ ppm with coupling constants $J_{2,3}$ $\sim 3.5 \mathrm{~Hz}$ ); the same protons of 2,5-FDCA occur at $7.28 \mathrm{ppm}$ as a singlet (DMSO- $d_{6}$ ). Asymmetric structures of the monoamides $\mathbf{5 a - d}$ were confirmed by observation of two distinct signals for the carboxyl and amide groups.

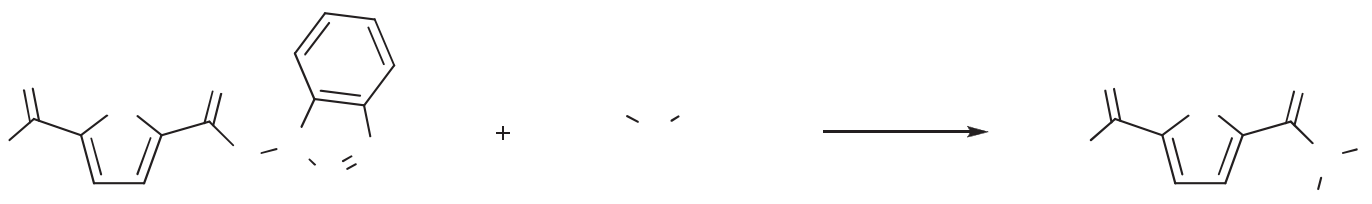

Scheme 3. Synthesis of furan-2,5-dicarboxylic acid monoamides 5a-d.

Regarding consecutive amidation: we did not isolate significant amounts of the symmetrical 2,5-FDCA diamides. Less polar fractions usually contained traces of such by-products, detected by HPLC-MS, but never in isolable yields.

\section{Conclusions}

In conclusion, we successfully achieved regioselective monoamidation of 2,5-FDCA 1 using TBTU (2). We found that excellent regioselectivity in forming the activated monobenzotriazoyl ester $\mathbf{3}$ was achieved by dropwise addition of a TBTU solution in DMF into a dilute solution of $\mathbf{1}$ in the same solvent containing DIPEA at ambient temperature. The use of 3.5 equiv DIPEA and 1.2 equiv. of TBTU is important for complete conversion of the substrate $\mathbf{1}$. Devided crude reaction mixture was used directly in the subsequent coupling reactions with benzylamine, 
diethylamine, piperidine and aniline, respectively. Four representative 2,5-FDCA monoamides 5a-d were prepared and isolated in yields of 73-82 \%. Traces of symmetric 2,5-FDCA diamides were detected in the less polar chromatographic fractions, but never in isolable yields.

\section{Experimental Section}

General. All melting points were measured on a Melting Point B-540 apparatus (Büchi). NMR spectra were recorded on a Varian VRX-300 spectrometer $\left({ }^{1} \mathrm{H}, 300 \mathrm{MHz}\right.$ and $\left.{ }^{13} \mathrm{C}, 75 \mathrm{MHz}\right)$ in methanol-d $\mathrm{d}_{4}$. Infrared (IR) spectra were recorded on a Nicolet 5700 FT-IR spectrometer with ATR Smart Orbit Diamond adapter (Thermo Electron Corporation). HRMS analyses were performed on an Orbittrap Velos Pro spectrometer (H-ESI) in positive mode (Thermo Fisher Scientific). TLC analyses were carried out using TLC Silica gel $60 \mathrm{~F}_{254}$ (aluminium sheets, Merck) and visualized by UV light or by permanganate solution followed by heating. Flash column chromatography was performed on a Büchi system (Pump Manager C-615 and Fraction Collector C-660) using Normasil 60 silica gel (0.040-0.063 mm) (VWR). All solvents were dried and distilled according to conventional methods. All reagents were purchased from Aldrich, Acros Organics, Alfa-Aesar, Merck and Mikrochem Trade and were used without further purification.

5-[(1H-1,2,3-Benzotriazol-1-yloxy)carbonyl]furan-2-carboxylic acid (3). To a stirred solution of 2,5-FDCA 1 (1.2 g, $7.69 \mathrm{mmol}, 1$ equiv) in anhydrous DMF (32 mL) was added DIPEA (3.54 $\mathrm{mL}, 26.92$ mmol, 3.5 equiv.), the reaction flask was flushed with argon, and the solution of TBTU 2 (2.96 g, $9.23 \mathrm{mmol}, 1.2$ equiv) in anhydrous DMF (18 mL) was added dropwise at ambient temperature over one hour. After the mixture was stirred for an additional hour, the solution of $\mathbf{3}$ was divided in four equal portions, and each of them was used in the next reaction with a corresponding amine.

General synthetic procedure, exemplified by 5-(benzylcarbamoyl)furan-2-carboxylic acid (5a). To a DMF solution of activated monobenzotriazoyl furoate $\mathbf{3}$ from the previous reaction (1.92 mmol, 1 equiv), benzylamine 4a $(411 \mathrm{mg}, 419 \mu \mathrm{L}, 3.84 \mathrm{mmol}, 2$ equiv.) was added in one portion and the mixture was stirred at r.t. for 2 hours under argon atmosphere. Afterwards the mixture was cooled in an ice-bath, $\mathrm{HCl}(2 \mathrm{~N}, 10 \mathrm{~mL})$ was added and the product was extracted with ethyl acetate $(3 \times 15 \mathrm{~mL})$. The combined organic layers were washed with brine, dried over $\mathrm{MgSO}_{4}$, and the solvent was evaporated under reduced pressure. The product was isolated by flash column chromatography $\left(\mathrm{CH}_{2} \mathrm{Cl}_{2} / \mathrm{CH}_{3} \mathrm{OH} / \mathrm{CH}_{3} \mathrm{COOH}, 7: 1: 0.1\right)$.

5-(Benzylcarbamoyl)furan-2-carboxylic acid (5a). Slightly yellow solid, yield 75\%, $355 \mathrm{mg}$, $\mathrm{R}_{f}=0.13\left(\mathrm{CH}_{2} \mathrm{Cl}_{2} / \mathrm{CH}_{3} \mathrm{OH} / \mathrm{CH}_{3} \mathrm{COOH}, 7: 1: 0.1\right), \mathrm{mp} 130-134{ }^{\circ} \mathrm{C}$; IR $\left(v_{\max }, \mathrm{cm}^{-1}\right): 3392,3292$, 2929, 1731, 1637, 1590, 1496, 1383, 1295, 1245, 1217, 720, 695. ${ }^{1} \mathrm{H}$ NMR (300 MHz): $\delta_{\mathrm{H}} 5.48$ $(2 \mathrm{H}, \mathrm{s}), 8.01(1 \mathrm{H}, \mathrm{d}, J 3.4 \mathrm{~Hz}), 8.06(1 \mathrm{H}, \mathrm{d}, J 3.4 \mathrm{~Hz}), 8.15-8.40(5 \mathrm{H}, \mathrm{m}) .{ }^{13} \mathrm{C}$ NMR $(75 \mathrm{MHz})$ : $\delta_{\mathrm{C}} 44.0\left(\mathrm{CH}_{2}\right), 116.1(\mathrm{CH}), 117.2(\mathrm{CH}), 128.4(\mathrm{CH}), 128.8(\mathrm{CH}), 129.6(\mathrm{CH}), 139.5(\mathrm{C}), 149.5$ 
(C), 151.1 (C), 160.3 (C), 164.6 (C); HRMS (ESI): calcd for $\mathrm{C}_{13} \mathrm{H}_{12} \mathrm{NO}_{4}[\mathrm{M}+\mathrm{H}]^{+}$: 246.0766 , found 246.0760 .

5-(Diethylcarbamoyl)furan-2-carboxylic acid (5b). Colourless solid, yield 77\%, $310 \mathrm{mg}, \mathrm{R}_{f}=$ $0.18\left(\mathrm{CH}_{2} \mathrm{Cl}_{2} / \mathrm{CH}_{3} \mathrm{OH} / \mathrm{CH}_{3} \mathrm{COOH}, 7: 1: 0.1\right)$, mp 145-150 ${ }^{\circ} \mathrm{C}$; IR $\left(v_{\max }, \mathrm{cm}^{-1}\right): 3429,3390,3109$, $2978,2873,1716,1597,1568,1432,1382,1290,1212,821,789 .{ }^{1} \mathrm{H}$ NMR $(300 \mathrm{MHz}): \delta_{\mathrm{H}} 2.10-$ $2.35(6 \mathrm{H}, \mathrm{m}), 4.40-4.70(4 \mathrm{H}, \mathrm{m}), 7.99(1 \mathrm{H}, \mathrm{d}, J 3.6 \mathrm{~Hz}), 8.09(1 \mathrm{H}, \mathrm{d}, J 3.6 \mathrm{~Hz}) .{ }^{13} \mathrm{C}$ NMR $(75$ $\mathrm{MHz}): \delta_{\mathrm{C}} 13.0\left(\mathrm{CH}_{3}\right), 15.0\left(\mathrm{CH}_{3}\right), 42.8\left(\mathrm{CH}_{2}\right), 44.5\left(\mathrm{CH}_{2}\right), 117.6(\mathrm{CH}), 117.7(\mathrm{CH}), 149.7(\mathrm{C})$, 150.3 (C), 161.1 (C), 163.3 (C); HRMS (ESI): calcd for $\mathrm{C}_{10} \mathrm{H}_{14} \mathrm{NO}_{4}[\mathrm{M}+\mathrm{H}]^{+}: 212.0923$, found 212.0916.

5-(Piperidine-1-carbonyl)furan-2-carboxylic acid (5c). Colourless solid, yield 82\%, $350 \mathrm{mg}$, $\mathrm{R}_{f}=0.16\left(\mathrm{CH}_{2} \mathrm{Cl}_{2} / \mathrm{CH}_{3} \mathrm{OH} / \mathrm{CH}_{3} \mathrm{COOH}, 7: 1: 0.1\right), \mathrm{mp} 110-112{ }^{\circ} \mathrm{C}$; IR $\left(v_{\max }, \mathrm{cm}^{-1}\right): 3390,3130$, 2937, 2856, 1727, 1596, 1567, 1434, 1385, 1275, 1217, 787, 750. ${ }^{1} \mathrm{H}$ NMR $(300 \mathrm{MHz}): \delta_{\mathrm{H}} 2.54-$ $2.72(6 \mathrm{H}, \mathrm{m}), 4.50-4.85(4 \mathrm{H}, \mathrm{m}), 7.93(1 \mathrm{H}, \mathrm{d}, J 3.5 \mathrm{~Hz}), 8.07(1 \mathrm{H}, \mathrm{d}, J 3.5 \mathrm{~Hz}) .{ }^{13} \mathrm{C} \mathrm{NMR}(75$ $\mathrm{MHz}): \delta_{\mathrm{C}} 26.9\left(\mathrm{CH}_{2}\right), 27.8\left(\mathrm{CH}_{2}\right), 45.2\left(\mathrm{CH}_{2}\right), 117.5(\mathrm{CH}), 117.6(\mathrm{CH}), 149.8(\mathrm{C}), 150.0(\mathrm{C})$, 160.5 (C), 163.6 (C); HRMS (ESI): calcd for $\mathrm{C}_{11} \mathrm{H}_{14} \mathrm{NO}_{4}[\mathrm{M}+\mathrm{H}]^{+}: 224.0923$, found 224.0916.

5-(Phenylcarbamoyl)furan-2-carboxylic acid (5d). Slightly yellow solid, yield 73\%, $325 \mathrm{mg}$, $\mathrm{R}_{f}=0.13\left(\mathrm{CH}_{2} \mathrm{Cl}_{2} / \mathrm{CH}_{3} \mathrm{OH} / \mathrm{CH}_{3} \mathrm{COOH}, 7: 1: 0.1\right)$, decomp. $135{ }^{\circ} \mathrm{C}$ (gas formation); IR ( $v_{\max }$, $\left.\mathrm{cm}^{-1}\right): 3369,3292,3130,1725,1652,1599,1576,1552,1381,1322,1263,785,752,690 .{ }^{1} \mathrm{H}$ NMR (300 MHz): $\delta_{\mathrm{H}} 8.00(1 \mathrm{H}, \mathrm{d}, J 3.4 \mathrm{~Hz}), 8.06(1 \mathrm{H}, \mathrm{t}, J 7.4 \mathrm{~Hz}), 8.14(1 \mathrm{H}, \mathrm{d}, J 3.4 \mathrm{~Hz}), 8.28$ $(2 \mathrm{H}, \mathrm{t}, J 8.0 \mathrm{~Hz}), 8.64(2 \mathrm{H}, \mathrm{d}, J 8.0 \mathrm{~Hz}) \cdot{ }^{13} \mathrm{C}$ NMR $(75 \mathrm{MHz}): \delta_{\mathrm{C}} 116.4(\mathrm{CH}), 116.7(\mathrm{CH}), 121.5$ $(\mathrm{CH}), 125.7(\mathrm{CH}), 130.0(\mathrm{CH}), 139.3(\mathrm{C}), 148.8(\mathrm{C}), 152.4(\mathrm{C}), 158.3(\mathrm{C}), 165.9(\mathrm{C})$; HRMS (ESI): calcd for $\mathrm{C}_{12} \mathrm{H}_{10} \mathrm{NO}_{4}[\mathrm{M}+\mathrm{H}]^{+}:$232.0610, found 232.0604 .

\section{Acknowledgements}

This work was supported by the Slovak Research and Development Agency under contract No. APVV-0367-10.

\section{References}

1. Mao, H.; Genco, J. M.; Yoon, S.-H.; van Heiningen, A.; Pendse, H. J. Biobased Mat. Bioenergy 2008, 2, 177. http://dx.doi.org/10.1166/jbmb.2008.309.

2. Carvalheiro, F.; Duarte, L. C.; Gírio, F. M. J. Sci. Ind. Res. 2008, 67, 849.

3. van Heiningen, A. Pulp and Paper Canada 2006, 107, 38.

4. Lewkowski, J. Arkivoc 2001, (i), 17.

5. Bozell, J. J.; Petersen, G. R. Green Chem. 2010, 12, 539. http://dx.doi.org/10.1039/B922014C. 
6. Brasholz, M.; von Känel, K.; Hornung, C. H.; Saubern, S.; Tsanaktsidis, J. Green Chem. 2011, 13, 1114. http://dx.doi.org/10.1039/C1GC15107J.

7. Gandini, A.; Belgacem, M. N. Prog. Polym. Sci. 1997, 22, 1203. http://dx.doi.org/10.1016/S0079-6700(97)00004-X.

8. Gomes, M.; Gandini, A.; Silvestre, A. J. D.; Reis, B. J. Polym. Sci. Part A: Polym. Chem. 2011, 49, 3759. http://dx.doi.org/10.1002/pola.24812.

9. Gubbels, E.; Jasinska-Walc, L.; Koning, C. E. J. Polym. Sci. Part A: Polym. Chem. 2013, 51, 890.

http://dx.doi.org/10.1002/pola.26446.

10. Kovash, C. S. Jr; Pavlacky, E,; Selvakumar, S.; Sibi, M. P.; Webster, D. C. ChemSusChem 2014, 7, 2289. http://dx.doi.org/10.1002/cssc.201402091.

11. Lewkowski, J. Pol. J. Chem. 2001, 75, 1943.

12. Koopman, F.; Wierckx, N.; de Winde, J. H.; Ruijssenaars, H. J. Bioresour. Technol. 2010 , $107,6291$. http://dx.doi.org/10.1016/j.biortech.2010.03.050.

13. Partenheimer, W.; Grushin, V. V. Adv. Synth. Catal. 2001, 343, 102. http://dx.doi.org/10.1002/1615-4169(20010129)343:1<102::AID-ADSC102>3.0.CO;2-Q.

14. Pan, T.; Deng, J.; Xu, Q.; Zuo, Y.; Guo, Q.-X.; Fu, Y. ChemSusChem. 2013, 6, 47. http://dx.doi.org/10.1002/cssc.201200652.

15. Thiyagarajan, S.; Pukin, A.; Van Haveren, J.; Lutz, M.; Van Es, D. S. RSC Advances 2013, 3, 15678. http://dx.doi.org/10.1039/c3ra42457j.

16. Richter, O.; Franke, O. WO Patent 2012101015(A1) (2012, Sued Chemie AG.)

17. Casanova, O.; Iborra, S.; Corma, A. J. Catal. 2009, 265, 109. http://dx.doi.org/10.1016/j.jcat.2009.04.019.

18. Menegazzo, F.; Fantinel, T.; Signoretto, M.; Pinna, F.; Manzoli, M. J. Catal. 2014, 319, 61. http://dx.doi.org/10.1016/j.jcat.2014.07.017.

19. Khusnutdinov, R. I.; Bayguzina, A. R.; Mukminov, R. R. Russ. Chem. Bull. 2013, 62, 93, and references cited therein. [Izv. Akad. Nauk, Ser. Khim. 2013, 62, 93.]. http://dx.doi.org/10.1007/s11172-013-0012-6.

20. Citron, C. A.; Rabe, P.; Dickschat, J. S. J. Nat. Prod. 2012, 75, 1765. http://dx.doi.org/10.1021/np300468h.

21. Fischer, R.; Fišerová, M. Arkivoc 2013, (iv), 405. http://dx.doi.org/10.3998/ark.5550190.p008.285

22. Munoz de Diego, C.; Schammel, W. P.; Dam, M. A.; Gruter, G. J. M. WO Patent 2011043660(A2) (2011, Furanix Technologies B.V.) 
23. Mazoyer, E.; De Sousa Dias, A. S. V.; Mckay, B.; Baars, H. J.; Vreeken, V. P. C.; Gruter, G. J. M.; Sikkenga, D. L. WO Patent 2014/163500A1 (2014, Furanix Technologies B.V.)

24. Sanborn, A. WO Patent 2010132740(A2) (2010, Archer Daniels Midland Company.)

25. Schmuck, C.; Machon, U. Eur. J. Org. Chem. 2006, 4385. http://dx.doi.org/10.1002/ejoc.200600324.

26. Stensrud, K.; Venkitasubramanian, P. WO Patent 2014099438(A2) (2014, Archer Daniels Midland Company.)

27. Racané, L.; Tralić-Kulenović, V.; Kraljević Pavelić, S.; Ratkaj, I.; Peixoto, P.; Nhili, R.; Depauw, S.; Hildebrand, M.-P.; David-Cordonnier, M.-H.; Pavelić, K.; Karminski-Zamola, G. J. Med. Chem. 2010, 53, 2418. http://dx.doi.org/10.1021/jm901441b.

28. Patch, R. J.; Brandt, B. M.; Asgari, D.; Baindur, N.; Chadha, N. K.; Georgiadis, T.; Cheung, W. S.; Petrounia, I. P.; Donatelli, R. R.; Chaikin, M. A.; Player, M. R. Bioorg. Med. Chem. Letters 2007, 17, 6070. http://dx.doi.org/10.1016/j.bmcl.2007.09.057.

29. James, L. I.; Korboukh, V. K.; Krichevsky, L.; Baughman, B. M.; Herold, J. M.; Norris, J. L.; Jin, J.; Kireev, D. B.; Janzen, W. P.; Arrowsmith, C. H.; Frye, S. V. J. Med. Chem. 2013, 56,7358 . http://dx.doi.org/10.1021/jm400919p.

30. Jarak, I.; Marjanović, M.; Piantanida, I.; Kralj, M.; Karminski-Zamola, G. Eur. J. Med. Chem. 2011, 46, 2807. http://dx.doi.org/10.1016/j.ejmech.2011.04.001.

31. Benecke, H. P.; Garbark, D. B.; Kawczak, A. W.; Clingerman, M. C. US Patent 2012316307(A1), (2012, Battelle Memorial Institute).

32. Friis, S. D.; Skrydstrup, T.; Buchwald, S. L. Org. Lett. 2014, 16, 4296. http://dx.doi.org/10.1021/o1502014b.

33. Knorr, R.; Trzeciak, A.; Bannwarth, W.; Gillessen, D. Tetrahedron Lett. 1989, 30, 1927. http://dx.doi.org/10.1016/S0040-4039(00)99616-3.

34. Bartkovitz, D. J.; Chu, X.-J.; Liu, J.-J.; Morgan, R. T.; Zhang, Z. US Patent 2011086854(A1) (2011, Hoffmann-La Roche Inc.)

35. Yu, T.; Belanger, D. B.; Kerekes, A. D.; Meng, Z.; Tagat, J. R.; Esposite, S. J.; Mandal, A. K.; Xiao, Y.; Kulkarni, B. A.; Zhang, Y.; Curran, P. J.; Doll, R.; Siddiqui, M. A. WO Patent 2008156614(A2) (2008, Schering Corp

36. Hamada, Y.; Ohta, H.; Miyamoto, N.; Yamaguchi, R.; Yamani, A.; Hidaka, K.; Kimura, T.; Saito, K.; Hayashi, Y.; Ishiura, S.; Kiso, Y. Bioorg. Med. Chem. Letters 2008, 18, 1654. http://dx.doi.org/10.1016/j.bmcl.2008.01.056.

37. Chen, X.; Zhan, P.; Pannecouque, C.; Balzarini, J.; De Clercq, E.; Liu, X. Eur. J. Med. Chem. 2012, 51, 60 . http://dx.doi.org/10.1016/j.ejmech.2012.02.019. 
38. Carpino, L. A.; Ionescu, D.; El-Faham, A. J. Org. Chem. 1996, 61, 2460. : and references cited therein.

http://dx.doi.org/10.1021/jo950912x. 